\title{
Usabilidad de Publicidad en el entorno móvil
}

\author{
Víctor Hugo Ríos Leyva ${ }^{1}$ y Leticia Amalia Neira Tovar ${ }^{2}$ \\ ${ }^{1}$ Universidad Autónoma de Nuevo León, México,victor.riosly@uanl.edu.mx +528110462913 \\ ${ }^{2}$ Universidad Autónoma de Nuevo León, México, letica.neiratv@uanl.edu.mx, +528183095219
}

\begin{abstract}
Información del artículo revisado por pares
Fecha de aceptación: junio-2021

Fecha de publicación en línea: diciembre-2021

DOI: https://doi.org/10.29105/vtga7.1-131
\end{abstract}

\begin{abstract}
Resumen
Existen diversos estudios que permiten analizar el efecto que tiene la publicidad dentro de las redes sociales en los dispositivos móviles, la mayoría de estos estudios se basan en cuestionarios de percepción y no dentro de las pruebas biométricas para el uso de la publicidad, este estudio se enfoca en utilizar el método de eye tracking para el estudio de la interacción humano-computador. Con el eye tracking los investigadores académicos utilizan información sobre el movimiento de los ojos y la mirada para evaluar los procesos de atención, comparar comportamientos de grupo y medir las respuestas visuales a los estímulos. Las principales marcas de consumo utilizan el seguimiento ocular para comprender mejor la experiencia del cliente y el rendimiento del producto al medir la atención visual a la información clave sobre la publicidad del producto, la marca y la personalización, así como el diseño de empaque. Debido a que el proceso de búsqueda está tan automatizado, este método es una herramienta atractiva para la investigación tanto cualitativa como cuantitativa, que permite a los investigadores aprovechar los procesos inconscientes determinados por nuestros sesgos y preferencias. Estas son 10 de las métricas de seguimiento ocular más comunes: Fijaciones y puntos de mirada, Mapas de calor, áreas de interés (ADI), Tiempo de primera fijación, Tiempo invertido, Relación, Secuencias de fijación, Revisitas, Primera duración de fijación y Duración media de la fijación. El seguimiento ocular es una métrica relativamente simple en
\end{abstract}

\section{Abstract}

There are several studies that allow to analyze the effect that advertising has within social networks on mobile devices, most of these studies are based on perception questionnaires and not within biometric tests for the use of advertising, this study focuses on using the eye tracking method for the study of human-computer interaction. With eye tracking, academic researchers use information about eye and eye movement to evaluate attention processes, compare group behaviors, and measure visual responses to stimuli. Leading consumer brands use eye tracking to better understand customer experience and product performance by measuring visual attention to key information about product advertising, branding and customization, as well as packaging design. Because the search process is so automated, this method is an attractive tool for both qualitative and quantitative research, allowing researchers to take advantage of the unconscious processes determined by our biases and preferences. These are 10 of the most common eye tracking metrics: Fixations and Lookpoints, Heat Maps, Areas of Interest (AOI), First Fixation Time, Time Spent, Ratio, Fixation Sequences, Visites, First Fixation Duration, and Average Fixation Duration. Eye tracking is a relatively simple metric in many ways; the hard part is knowing how to process the given data. 
muchos sentidos; la parte difícil es saber cómo procesar los datos que proporciona.

Palabras clave: Publicidad conductual en línea, publicidad, usabilidad, redes sociales, seguimiento ocular.

\section{INTRODUCCIÓN}

En los últimos años, la Comisión Federal de Comercio (FTC) de EE. UU. Ha expresado su preocupación sobre cómo las practicas de publicidad conductual en línea (Online Behavioral Advertising - OBA, por sus siglas en inglés) afectan la privacidad del consumidor en el entorno de tecnología celular (FTC, 2013, 2017). El enfoque de OBA es enviar anuncios dirigidos a los consumidores en función de los datos recopilados mediante el seguimiento de sus actividades en línea a lo largo del tiempo en todos los dispositivos. La industria de la publicidad en línea se autorregula en gran medida, pero ha ajustado su código de autorregulación en respuesta a los problemas de privacidad planteados por la FTC (DAA, 2013, 2015); (NAI, 2015a,b,c). Esta investigación examina nuevas herramientas creadas para el entorno celular como parte del modelo de selección y notificación de la industria, que pueden crear anuncios de comportamiento para los consumidores y proporcionar herramientas de ajuste de preferencias.

Estudios anteriores han encontrado que los modelos de notificación y selección tienen importantes problemas de usabilidad (Leon et al., 2012); (Hastak and Culnan, 2010). Las organizaciones de protección de los derechos del consumidor (Gellman and Dixon, 2011), investigadores académicos, eruditos legales (Barocas and Nissenbaum, 2009), (Hoofnagle et al., 2012), (Brotherton, 2011), (Solove, 2007), (Nissenbaum, 2011) y la FTC han sido ampliamente criticados por no resolver adecuadamente los problemas de privacidad en línea de los consumidores. La útil discusión de Bennett sobre la regulación de OBA (Bennett, 2010) describe el desarrollo legal del sistema de políticas de autorregulación para 2010. Esta política se originó en la audiencia pública en curso de la
Keywords: OBA (Online Behavioral Advertising), advertising, usability, social networks, eye tracking. JEL: M31 Marketing y M37 Publicidad, O32 Gestión de la innovación tecnológica y de la I + D

FTC e hizo recomendaciones a la industria, respondiendo, formulando y ajustando sus reglas de autorregulación.

Se cree que los anunciantes utilizan la tecnología para superar la elección del consumidor (Hoofnagle et al., 2012). La industria afirma que la ventaja de OBA es que las personas necesitan personalización e individualización. Sin embargo, debido a que la industria, no el consumidor, toma decisiones personalizadas, el control del consumidor es una fachada (Turow, 2012). Esta asimetría de información entre consumidores y empresas pone a los consumidores en desventaja (Li, 2012); (FTC, 2007). Los estadounidenses se preocupan por la privacidad de los datos. El Índice de Privacidad del Consumidor de EE. UU. (TRUSTe/NCSA, 2016) encontró que el $92 \%$ de los usuarios de Internet están preocupados por la privacidad en línea, mientras que el 74 $\%$ de los usuarios declararon haber restringido las actividades en línea por la siguiente razón: están preocupados por su privacidad.

En el 2019 la compañía Marketing 4 Ecommerce en México (https://marketing4ecommerce.mx/)

comentó que la polémica que se ha desatado en torno a la privacidad de los datos en redes sociales, encabezada por Facebook en el caso Cambridge Analytica, ha desencadenado ansiedad y preocupación sobre la distribución y la forma en que se utilizan los datos personales en internet. De acuerdo con el reciente Informe Global sobre la Privacidad de Kaspersky, un tercio de los usuarios mexicanos (32\%) estarían dispuestos a darse de baja de las redes sociales sin con ello pudieran garantizar su privacidad digital.

De hecho, el informe revela que el 13 $\%$ de los mexicanos reconoció ceder información personal con el fin de registrarse o participar en juegos y concursos en redes sociales. De forma adicional, si estos mexicanos abandonarán las redes sociales, 
saben que se haría más complejo el ingreso a diversas páginas y servicios web, ya que el $66 \%$ de los mexicanos vincula estas cuentas a perfiles de Twitter o Facebook. Si bien en la actualidad las redes sociales han llegado al punto en que la calidad de la experiencia de usuario es directamente proporcional a la cantidad de datos personales que los usuarios estén dispuestos a ceder, como es la información financiera, de geolocalización, patrones de compra y hasta situación sentimental, no sería suficiente abandonar por completo este tipo de plataformas para proteger la privacidad digital de todos ellos.

Esta investigación ha contribuido al crecimiento de la literatura sobre la disponibilidad y eficacia de la autorregulación de los mecanismos de elección de privacidad.

Más adelante hablaremos de la autorregulación de la industria, el sistema Eye tracking para el estudio de objetivos, la metodología de esta investigación, así como los resultados de esta.

\section{MARCO TEÓRICO}

En los Estados Unidos, existen pocas regulaciones formales para controlar la publicidad conductual en línea. A excepción de ciertas leyes federales que protegen los datos médicos, financieros y de datos de los niños, la OBA está sujeta a la autorregulación de la industria y a la supervisión federal limitada. En el papel de proteger a los consumidores, la FTC brindó orientación a la industria de la publicidad en Internet basándose en audiencias públicas (FTC, 2009, 2012, 2013) e investigó las quejas.

$\mathrm{La}$ industria ha respondido con la esperanza de evitar la promulgación de una legislación formal.

\subsection{Autorregulación de la industria}

La Digital Advertising Alliance (DAA) es la organización autorreguladora general de la publicidad en línea en los Estados Unidos. La Network Advertising Initiative (NAI), que se centra en los anunciantes externos, también ha sido indispensable en el desarrollo de este marco. La DAA añadió tres documentos sobre la base de principios (DAA, 2009), proporcionando a las miembros recomendaciones sobre el uso de datos en varios sitios (DAA, 2011), el entorno móvil (DAA, 2013) y el uso de datos en varios dispositivos (DAA, 2015). NAI actualizó su Código de conducta de autorregulación (NAI, 2008, 2013, 2015c).

Los códigos DAA y NAI requieren la notificación de la recopilación de datos en la política de privacidad del sitio web. Muchos estudios han demostrado que estas estrategias son demasiado complicadas, tediosas y raras veces leídas (McDonald and Cranor, 2008); (Cranor, 2012; Komanduri et al., 2011; Schwaig et al., 2006), especialmente en un entorno de tecnología celular Singh et al. (2011). Además, aunque resuelven el seguimiento basado en cookies, recientemente han comenzado a abordar otras formas de tecnología de seguimiento (Hoofnagle et al., 2012); (Mayer and Mitchell, 2012); (Acar et al., 2014; Ayenson et al., 2011); (Kuehn and Mueller, 2012). NAI emitió una guía técnica sin cookies (NAI, 2015b); DAA comenzó a abordar este requisito (DAA, 2017).

El código también requiere que los consumidores opten por no participar (o recibir según la sensibilidad de la información) las opciones de publicidad basada en el comportamiento. Sin embargo, incluso si los consumidores deciden optar por no recibir "publicidad basada en intereses" (en términos de la industria), esto no significa que el seguimiento de los consumidores se detendrá.

Los defensores de la privacidad continúan planteando preocupaciones sobre la práctica de la OBA, y los investigadores han encontrado que las medidas de autorregulación de la industria son ineficaces (Leon et al., 2012; Hastak and Culnan, 2010), (Gellman and Dixon, 2011), (Cranor, 2012), y son insuficientes para proteger la privacidad del consumidor (King and Jessen, 2010a, 2010b). Muchos estudios académicos han encontrado que los consumidores tienen una comprensión limitada de la OBA y cómo elegir no recibir OBA (Leon et al., 2012a; Cranor, 2012b; Leon et al. 2012b). Incluso para aquellos que lo hacen, salir de OBA no significa necesariamente que puedan detener los archivos de configuración y monitoreo en 
línea a través de las prácticas de recopilación de datos de la industria, que es el problema más preocupante. $\mathrm{Y}$ el impacto en la privacidad del consumidor (Brotherton, 2012).

El modelo de notificación y elección de la industria publicitaria asume que los consumidores 1) notarán la información pública, 2) conocerán la información pública, 3) sabrán cómo podrán hacer clic en él, 4) para comprender la información proporcionada en la página vinculada y 5) para comprender cómo funciona el mecanismo de salida. Incluso entonces, cuando los usuarios solo pueden aplicarlo al sitio temporalmente, es posible que sigan pensando que están realizando cambios generales y permanentes. Además, la presentación visual de la información de salida es completamente inconsistente. Esta investigación demuestra que existen grandes diferencias entre cada sitio.

2.2. ¿Qué es el sistema de "Eye Tracking"? ¿Cual es su función?

El Eye Tracking es una solución técnica diseñada para extraer información de los usuarios analizando los movimientos de sus ojos. Un eye tracker es una herramienta para realizar esta operación, e incluye un monitor especial que emite rayos infrarrojos a los ojos de la persona que está viendo la imagen a analizar. Estos rayos siguen la dirección desde la pupila del usuario hasta el dispositivo, por lo que puede calcular con precisión lo que está mirando.

\section{3. ¿Qué información recopilamos al realizar el Eye Tracking? \\ - Un lugar donde se mira constantemente. \\ - Lo que acaba de llamar su atención. \\ - Qué intenciones tiene esta persona. \\ - El estado de ánimo de esa persona. \\ - La ubicación del valor para el cliente. \\ - Si la señal visual contenida en la red está efectivamente dirigida al cliente. \\ - Los clientes tienen la capacidad de encontrar la información que necesitan en anuncios de la Web.}

2.4. ¿Cuál es el uso de esta información?

- Mejorar la estructura del contenido.
- Mejorar la experiencia del usuario en la web.

- Guiar a los usuarios para que logren sus objetivos comerciales.

- Facilite el proceso que desea que realicen los usuarios.

- Consiga la marca en Internet.

- Mejorar la imagen de marca a través del sitio web.

Teniendo en cuenta los resultados que nos proporcionará este análisis, podemos sacar algunas conclusiones que afectarán al diseño y estructura de la publicidad en la web.

\section{5. Úselo como entrada o dispositivo interactivo}

Se utiliza como herramienta para la interfaz de evaluación objetiva.

Aunque la precisión del Eye Tracking como dispositivo de entrada está lejos de otros dispositivos como un ratón o teclado, puede tener muchas aplicaciones prácticas, como su uso en un entorno de realidad virtual o por usuarios con movilidad limitada. Además, incluso en determinadas operaciones (como la selección de objetos de la interfaz), la mirada puede ser más rápida que usar un mouse, como lo muestran (Sibert and Jacob, 2000).

Cuando se utiliza el Eye Tracking como herramienta de evaluación, la información extraída ya no requiere análisis y respuesta en tiempo real del sistema, sino que se registra a través de archivos de registro para su posterior análisis e interpretación. Este trabajo se centrará en la segunda aplicación, estudiando los problemas y ventajas de la tecnología en el contexto de la investigación de usabilidad.

\subsection{Representación de datos}

Para poder interpretar la gran cantidad de datos re- copilados durante las sesiones de eye tracking, algunos paquetes de software generan una serie de animaciones y representaciones que resumen gráficamente el comportamiento visual de un usuario o grupo de usuarios.

Al analizar el comportamiento visual del usuario por separado, se suele utilizar una representación animada de un determinado punto de la interfaz, lo que significa que el participante siempre se centra en dónde 
enfocar, y el pequeño trazo en forma de línea representa la mirada anterior, como visto en el ejemplo incluido en el artículo de (Usolab, 2007b).

Una representación estática alternativa, principalmente adecuada para el análisis aglomerado de los patrones de exploración visual de grupos de usuarios, son los 'heatmaps' o mapas de calor (Fig. 1). En estas representaciones, las zonas 'calientes' o de mayor intensidad señalan dónde han fijado los usuarios su atención con mayor frecuencia.

Todas estas representaciones gráficas son muy ilustrativas, no solo del responsable de su análisis y evaluación. De hecho, estas representaciones tienen grandes habilidades de comunicación para el cliente final porque permiten una "captura de pantalla" para demostrar que el usuario no ha navegado por la interfaz de una manera ordenada y predecible como mucha gente podría creer (Spool, 2006). En (Usolab, 2007a), cuestionaron si focalizar este tipo de comunicación no podría mejorar estas representaciones para hacerlas más comprensibles y efectivas.

\subsection{Interpretación de datos}

En la prueba de Eye Tracking, el primer paso del evaluador es definir el "'área de interés" para cada interfaz. Estas áreas de interés son utilizadas por evaluadores o equipos de diseño (Jacob and Karn, 2003), y lo que pretenden descubrir a través de las pruebas es si son visibles y significativas para los usuarios (Poole and Ball, 2005).

$\mathrm{Al}$ analizar las miradas relacionadas con el área de interés, hemos obtenido información más completa y fácil de entender, como una serie de miradas que miran continuamente la misma área de interés o el recorrido o secuencia del área definida en el campo visual.

Los datos registrados a través del Eye Tracking pueden decirnos dónde fija el usuario la atención visual y qué otras áreas o "regiones de interés" no se están notando.

Poole and Ball (2005) realizaron una extensa revisión de los diferentes indicadores propuestos en la literatura científica, describiendo sus métodos de medición y su significado. Aquí hay unos ejemplos:

- Número total de enlaces: cuanto mayor sea el número de enlaces, menor será la eficacia de búsqueda, lo que puede indicar un problema con el diseño de la interfaz (esquema de organización).

- Número de fijaciones en el área de interés: Cuantas más fijaciones, mayor importancia tiene para el usuario.

- Medir la duración de la fijación en el área de interés: cuanto mayor sea la duración, más difícil será explicar el contenido del área.

- La densidad espacial de los objetos fijos: cuando los objetos fijos se concentran en un área pequeña, la eficiencia de la búsqueda visual es mayor, y si el grado de dispersión es mayor, la eficiencia de la búsqueda es menor.

- Tiempo de primera fijación: cuanto menos tiempo dedique el usuario a fijar el área de interés por primera vez, mayor será la capacidad de los atributos gráficos del área para atraer la atención visual.

Aunque este tipo de medidas ayudan a explicar objetivamente la exploración visual de los participantes, todavía no son exhaustivas por las razones de ciertos patrones o comportamientos visuales. Una forma posible de obtener esta información es utilizar el método de "pensamiento" o "pensar en voz alta". El método implica pedirle a cada participante que describa verbalmente lo que está pensando, qué preguntas tiene, por qué debería realizar una acción o explorar visualmente una u otra área de interés durante la prueba.

\section{MÉTODO}

Dentro de esta metodología se utilizaron las siguientes herramientas tecnológicas, a continuación, su descripción y características:

- Gazepoint GP3 eye tracker. - Sistema de $60 \mathrm{~Hz}$. 
- Calibración de 5 o 9 puntos.

- Movimiento de $25 \mathrm{~cm}$ (horizontal) x $11 \mathrm{~cm}$ (vertical).

○ $\pm 15 \mathrm{~cm}$ de movimiento de profundidad.

○ Ultra portable - $320 \times 45 \times 40$ $\mathrm{mm}(145 \mathrm{~g})$.

- Compatible con pantallas de 26.o más pequeñas.

- Software Gazepoint Analysis UX Edition que incluye:

- Mapa de calor.

- Ruta de fijación de la mirada.

- Captura de pantalla / Imagen / Vídeo / Web Agregación de datos de usuario múltiple.

- Zonas dinámicas de interés (AOIs).

- Exportación de Imágenes, Vídeos y Estadísticas.

- Grabación de Thinkaloud Voice and Webcam.

- Equipo de computo.

$\begin{array}{ll}\circ & \text { Laptop Dell 15-5558 } \\ \circ & \text { Intel Core i7-5500U 5ta. Gen. } \\ \circ & \text { RAM 8Gb. } \\ \circ & \text { DD 1Tb. } \\ \circ & \text { Pantalla 15.6" } \\ \circ & \text { Windows } 8.1\end{array}$

En esta investigación se incluyó una serie de 12 imágenes de publicidad para realizar las pruebas de Eyetracking y un cuestionario de salida para obtener la apreciación de los participantes.

Los participantes del estudio fueron reclutados de boca en boca. La muestra de conveniencia fue construida por estudiantes universitarios de ambos géneros y que dicha actividad se realizara en el mismo equipo de computo.

Aunque no se puede promover una pequeña muestra, hay suficientes participantes para determinar los principales problemas de usabilidad. Se descubrió que probar cinco usuarios suele ser suficiente para encontrar la mayoría de los problemas, mientras que 15 usuarios pueden encontrar casi todos los problemas (Nielsen, 2000).

La realización de las pruebas fue de la siguiente manera:
A los participantes que completaran las pruebas del Eyetracking, que correspondían a la publicidad anunciada en las redes sociales mas utilizadas, contestaron preguntas dentro de las cuales se incluía: ¿La publicidad observada te parece atractiva?, ¿La publicidad del producto ofrecida es buena?, ¿La red social en la cual se presenta la publicidad te parece adecuada? ¿Se entienden cuáles son las características y beneficios que te brindan los productos ofrecidos en la publicidad?, etc.

Después las pruebas realizadas, los participantes completaron el cuestionario de salida, y proporcionaron una lista de recursos de información sobre la segmentación y el seguimiento del comportamiento en la prueba de Eye tracking, basándose en 4 diferentes tipos de características visuales aplicadas a las imágenes de las publicaciones entre las cuales están: Descripción del producto, Apoyo visual del producto, Precio y Distractores.

\section{RESULTADOS}

Primeramente, se realizaron las pruebas de Eyetracking sobre 12 imágenes de publicidad en las redes sociales más utilizadas.

Los resultados de las pruebas sobre dichas imágenes se muestran en la Tabla 1. "Imágenes de publicidad y aplicación de Eye Tracking", así como en la imagen Fig. 2 "Gráficas de características visuales de las imágenes de publicidad".

Los resultados obtenidos sobre las encuestas de salida y su representación mediante gráficas se muestran en la Tabla 2. "Resultados y Gráficas de la encuesta de salida". (Las abreviaciones en las gráficas se denotan de la siguiente manera: TDA=Totalmente de acuerdo, DA=De acuerdo, IND=Indiferente, $\quad \mathrm{DES}=\mathrm{En}$ desacuerdo y NS/NC=No Sé/No Contesto).

\section{CONCLUSIONES}

Este estudio intenta comprender el comportamiento visual del usuario mediante el análisis del movimiento de los ojos, utilizándolo como herramienta para la interfaz de evaluación objetiva sobre la publicidad en línea o en redes sociales, con el fin de detectar la manera correcta la formación de anuncios publicitarios; en 
estudios anteriores mediante esta herramienta se han descubierto puntos de impacto importantes (tanto positivos como negativos) con los usuarios al momento de visualizar publicidad. En futuras investigaciones se realizarán pruebas mas a fondo y con diversas métricas de la aplicación de Eye tracking. 


\section{REFERENCIAS}

Artículo científico

Acar, G., Eubank, C., Englehardt, S., Juarez, M., Narayanan, A., and Diaz, C. (2014). The web never forgets: Persistent tracking mechanisms in the wild. In Proceedings of the 2014 ACM SIGSAC Conference on Computer and Communications Security, pages 674-689.

Artículo científico

Ayenson, M. D., Wambach, D. J., Soltani, A., Good, N., and Hoofnagle, C. J. (2011). Flash cookies and privacy ii: Now with html5 and etag respawning. Available at SSRN 1898390.

Ponencias, Simposios y conferencias

Barocas, S. and Nissenbaum, H. (2009). On notice: The trouble with notice and consent. In Proceedings of the Engaging Data Forum: The First International Forum on the Application and Management of Personal Electronic Information.

Artículo científico

Bennett, S. C. (2010). Regulating online behavioral advertising. J. Marshall L. Rev., 44:899.

Artículo científico

Brotherton, E. A. (2011). Big brother gets a makeover: behavioral targeting and the third-party doctrine. Emory LJ, 61:555.

Artículo científico

Cranor, L. F. (2012). ¿Can users control online behavioral advertising effectively? IEEE Security and Privacy, 10(2):93-96.

Informes y reportes gubernamentales, institucionales o empresariales

DAA (2009). Self-regulatory principles for online behavioral advertising.

Informes y reportes gubernamentales, institucionales o empresariales

DAA (2011). Self-regulatory principles for online multisite data.

Informes y reportes gubernamentales, institucionales o empresariales

DAA (2013). Application of self- regulatory principles to the mobile environment.

Informes y reportes gubernamentales, institucionales o empresariales

DAA (2015). Application of the self- regulatory principles of transparency and control to data used across devices.

Informes y reportes gubernamentales, institucionales o empresariales

DAA (2017). NAI and DAA launch new version of choice tools for interest-based advertising.

Informes y reportes gubernamentales, institucionales o empresariales

FTC (2007). Online behavioral advertising: Moving the discussion forward to possible self-regulatory principles. Retrieved June, 14:2008.

Informes y reportes gubernamentales, institucionales o empresariales

FTC (2009). Self-regulatory principles for online behavioral advertising. Federal Trade Commission Staff Report, Washington, DC.

Informes y reportes gubernamentales, institucionales o empresariales

FTC (2012). Protecting consumer privacy in an era of rapid change. FTC report.

Informes y reportes gubernamentales, institucionales o empresariales

FTC (2013). Mobile privacy disclosures: Building trust through transparency: a federal trade commission staff report. Date accessed, 2.

Informes y reportes gubernamentales, institucionales o empresariales

FTC (2017). Cross-device tracking.

Artículo científico

Gellman, R. and Dixon, P. (2011). Many failures: A brief history of privacy self-regulation in the United States. In World Privacy Forum.

Artículo científico

Hastak, M. and Culnan, C. (2010). Future of privacy forum online behavioral advertising "icon" study. 
Artículo científico

Hoofnagle, C. J., Soltani, A., Good, N., and Wambach, D. J. (2012). Behavioral advertising: The offer you can't refuse. Harv. L. and Pol'y Rev., 6:273.

Artículo científico

Jacob, R. J. and Karn, K. S. (2003). Eye tracking in human-computer interaction and usability research: Ready to deliver the promises. In The mind's eye, pages 573-605. Elsevier.

Artículo científico

Komanduri, S., Shay, R., Norcie, G., Ur, B., and Cranor, L. (2011). Adchoices? compliance with online behavioral advertising notice and choice requirements (cmu-cylab-11-005).

Artículo científico

Kuehn, A. and Mueller, M. (2012). Profiling the profilers: deep packet inspection and behavioral advertising in Europe and the United States. Available at SSRN 2014181.

Artículo científico

Leon, P., Ur, B., Shay, R., Wang, Y., Balebako, R., and Cranor, L. (2012). Why Johnny can't opt out: A usability evaluation of tools to limit online behavioral advertising. In Proceedings of the SIGCHI Conference on Human Factors in Computing Systems, pages 589-598.

Artículo científico

Li, Y. (2012). Theories in online information privacy research: A critical review and an integrated framework. Decision support systems, 54(1):471-481.

Ponencias, Simposios y conferencias

Mayer, J. R. and Mitchell, J. C. (2012). Third-party web tracking: Policy and technology. In 2012 IEEE Symposium on Security and Privacy, pages 413-427. IEEE.

Artículo científico

McDonald, A. M. and Cranor, L. F. (2008). The cost of reading privacy policies. Isla, 4:543.

Informes y reportes gubernamentales, institucionales o empresariales

NAI (2008). '2008 NAI principles the network advertising initiative's self-regulatory code of conduct".

Informes y reportes gubernamentales, institucionales o empresariales

NAI (2013). NAI code of conduct. Network Advertising Initiative, 201.

Informes y reportes gubernamentales, institucionales o empresariales

NAI (2015a). 2015 update to the NAI code of conduct.

Informes y reportes gubernamentales, institucionales o empresariales

NAI (2015b). Guidance for NAI members: Use of non-cookie technologies for interest-based advertising consistent with the NAI code of conduct. Network Advertising Initiative.

Informes y reportes gubernamentales, institucionales o empresariales

NAI (2015c). Update to the NAI mobile application code. Network Advertising Initiative.

Libro en línea

Nielsen, J. (2000). Why you only need to test with 5 users. 2000. Jakob Nielsen's Alertbox. Available on line from www.useit.com/alertbox/20000319.html.

Artículo científico

Nissenbaum, H. (2011). A contextual approach to privacy online. Daedalus, 140(4):32-48.

Artículo científico

Poole, A. and Ball, L. J. (2005). Eye tracking in human-computer interaction and usability research: current status and future prospects, 2005. United Kingdom: Psychology Department, Lancaster University.

Artículo científico

Schwaig, K. S., Kane, G. C., and Storey, V. C. (2006). Compliance to the fair information practices: ¿How are the fortune 500 handling online privacy disclosures? Information and management, 43(7):805-820. 


\section{Artículo científico}

Sibert, L. E. and Jacob, R. J. (2000). Evaluation of eye gaze interaction. In Proceedings of the SIGCHI conference on Human Factors in Computing Systems, pages 281-288.

Artículo científico

Singh, R. I., Sumeeth, M., and Miller, J. (2011). Evaluating the readability of privacy policies in mobile environments. International Journal of Mobile Human Computer Interaction (IJMHCI), 3(1):55-78.

Artículo científico

Solove, D. J. (2007). I've got nothing to hide and other misunderstandings of privacy. San Diego L. Rev., 44:745.

Libro en línea

Spool, J. (2006). Eyetracking: Worth the expense. User Interface Engineering. http://www.uie.com/brainsparks/2006/06/13/eyetracking-worth-the-expense.

Informes y reportes gubernamentales, institucionales o empresariales

TRUSTe/NCSA (2016). Truste/NCSA consumer privacy infographic - us edition. https://staysafeonline.org/wp-content/uploads/2017/09/TRUSTe-National-Cyber-SecurityAlliance-U.S.-Consumer-Privacy-Index- 2016-Infographic.pdf.

Artículo científico

Turow, J. (2012). The daily you: How the new advertising industry is defining your identity and your worth. Yale University Press.

Informes y reportes gubernamentales, institucionales o empresariales

Usolab (2007a). La aportación del eyetracking en el sector de usabilidad. febrero de 2007. http://www.usolab.com/articulos/eyetracking-usabilidad-comunicacion.php.

Informes y reportes gubernamentales, institucionales o empresariales

Usolab (2007b). Tiempo de inicio de navegación: análisis con eyetracking. marzo de 2007. http://www.usolab.com/articulos/eyetracking-navegacion.php. 


\section{FIGURAS}

Figura 1 Heatmap (Mapa de Calor).

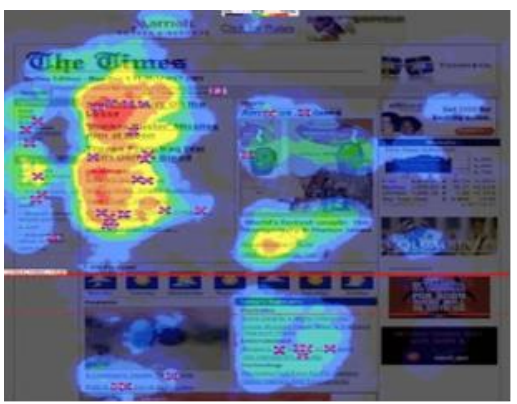

Figura 2. Gráficas de características visuales de las imágenes de publicidad.

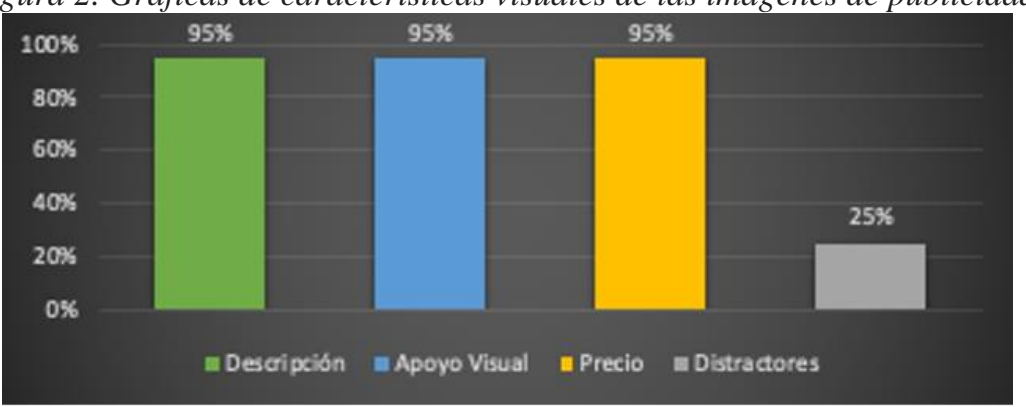

Tabla 1 Imágenes de publicidad y aplicación de Eye Tracking

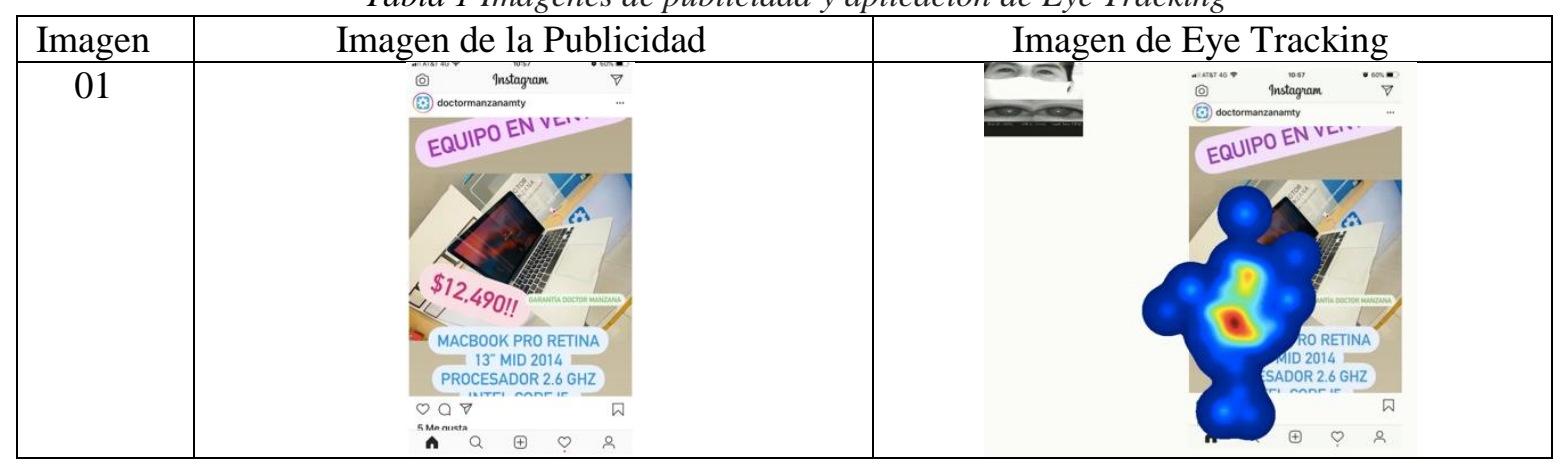




\begin{tabular}{|c|c|c|c|}
\hline 02 & 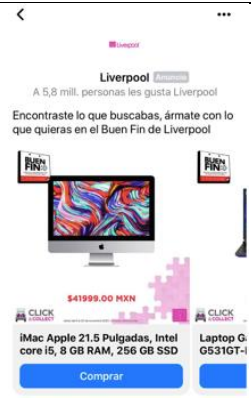 & $\begin{array}{ll}901 \\
\infty\end{array}$ & (3) \\
\hline 03 & 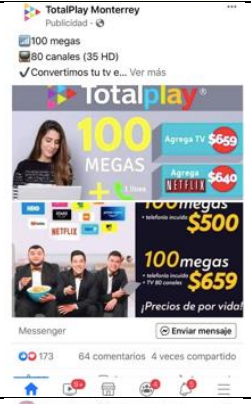 & कe & (1) \\
\hline 04 & 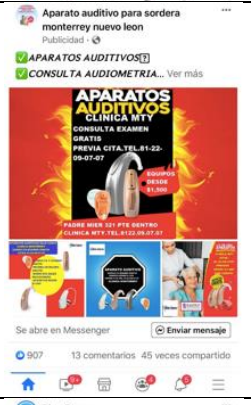 & 20 & 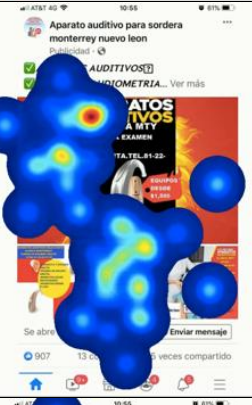 \\
\hline 05 & 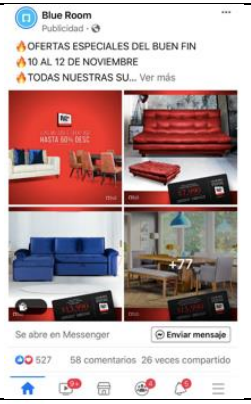 & & 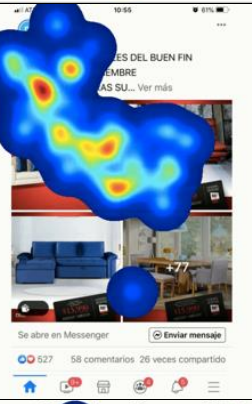 \\
\hline 06 & 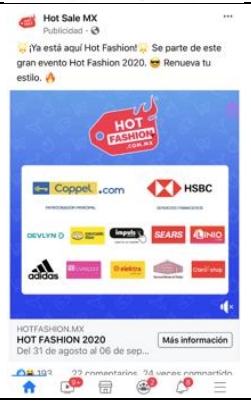 & & 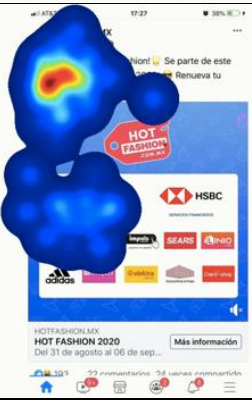 \\
\hline
\end{tabular}




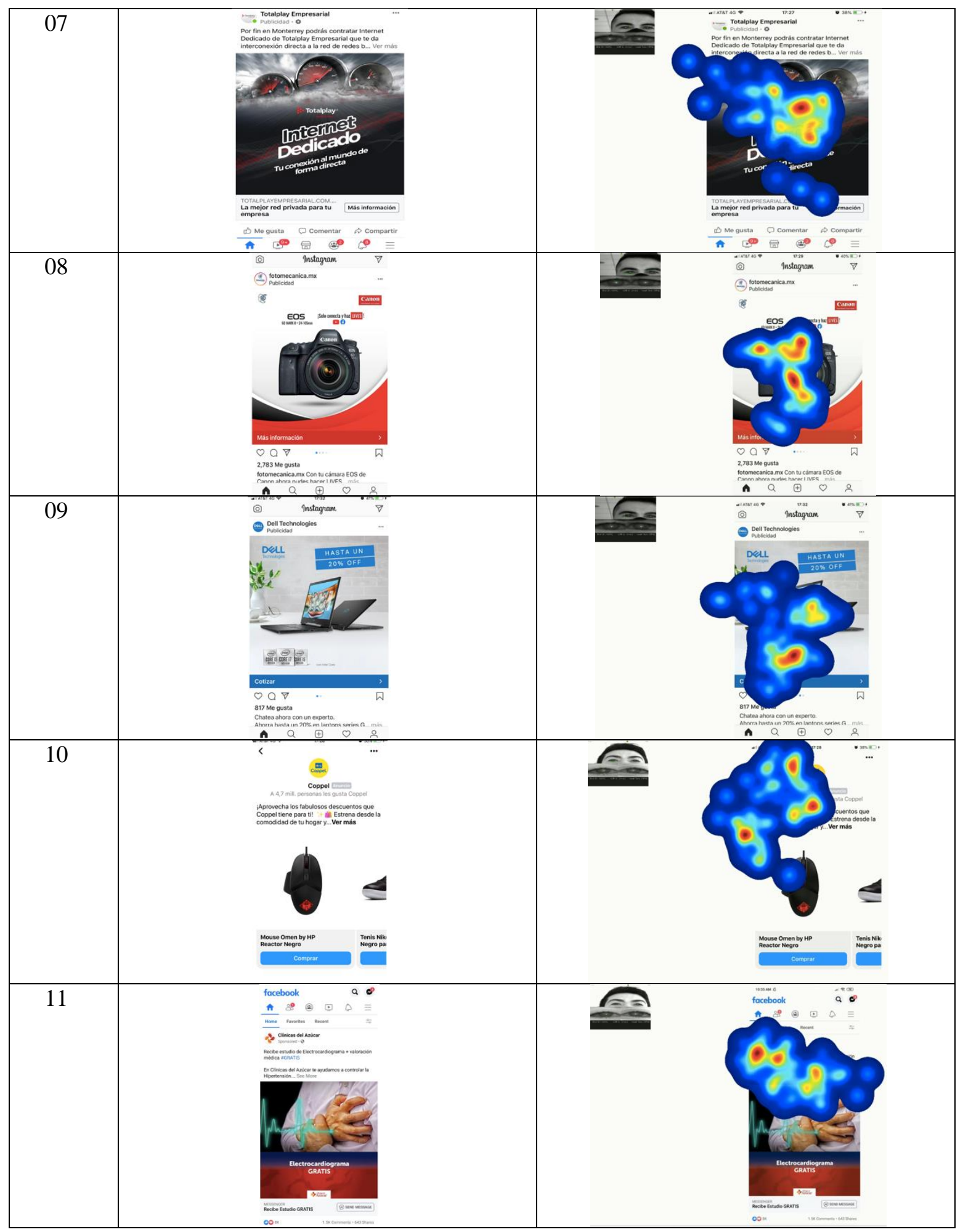




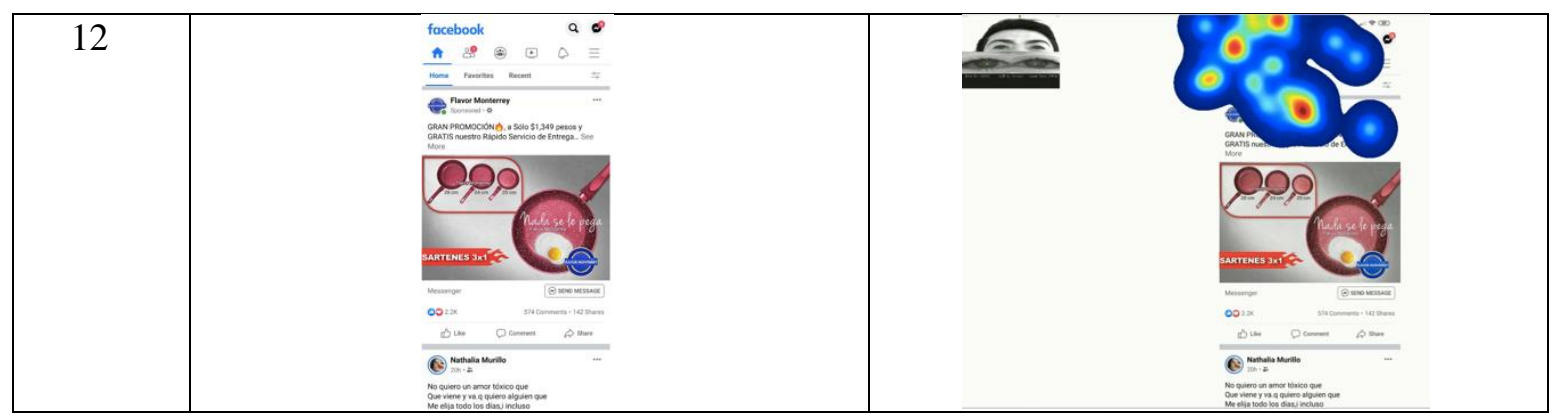

Tabla 2. Resultados y Gráficas de la encuesta de salida.

(Las abreviaciones en las gráficas se denotan de la siguiente manera: TDA=Totalmente de acuerdo, DA=De acuerdo, IND=Indiferente, DES=En desacuerdo y NS/NC=No Sé/No Contesto)

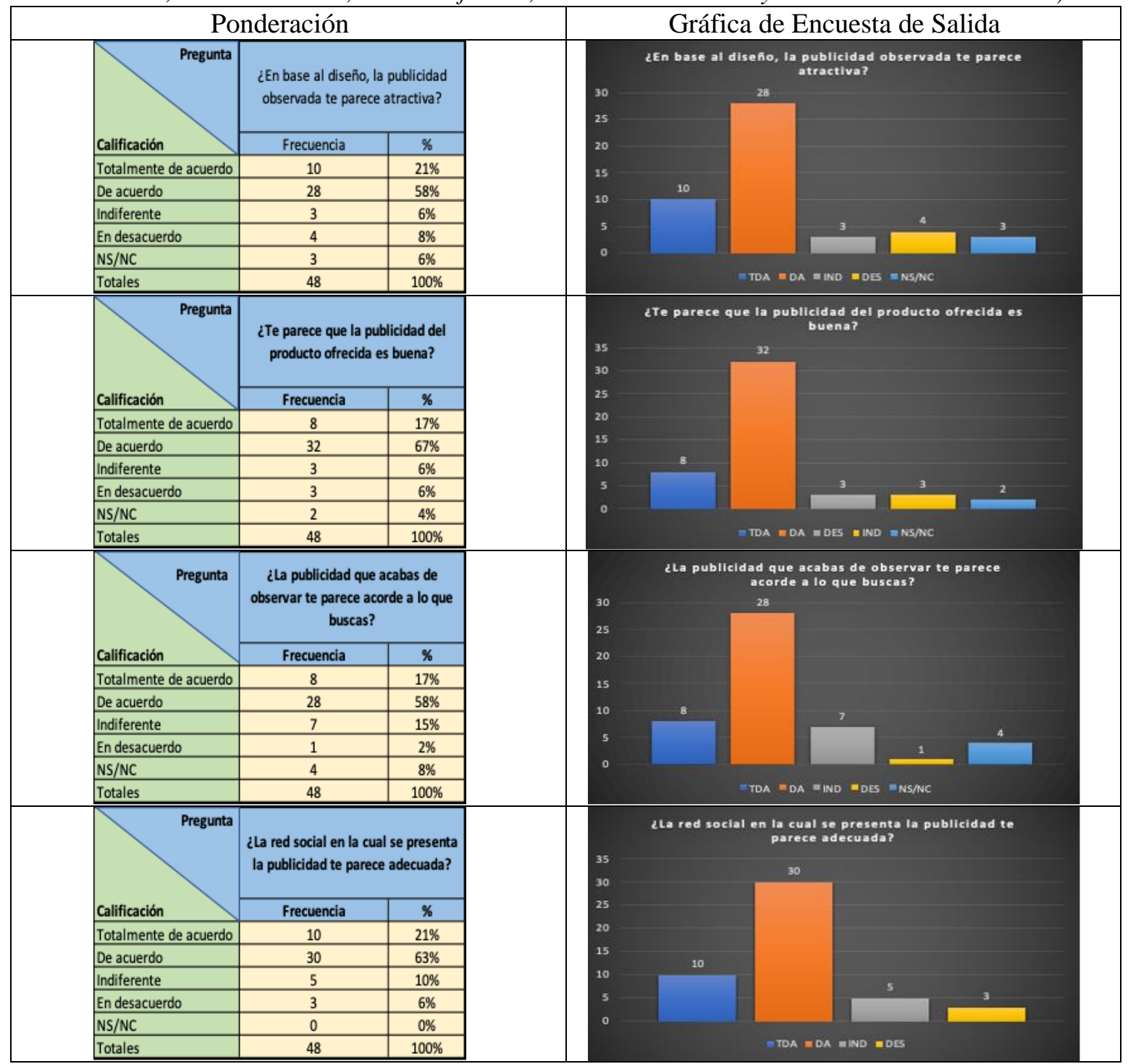




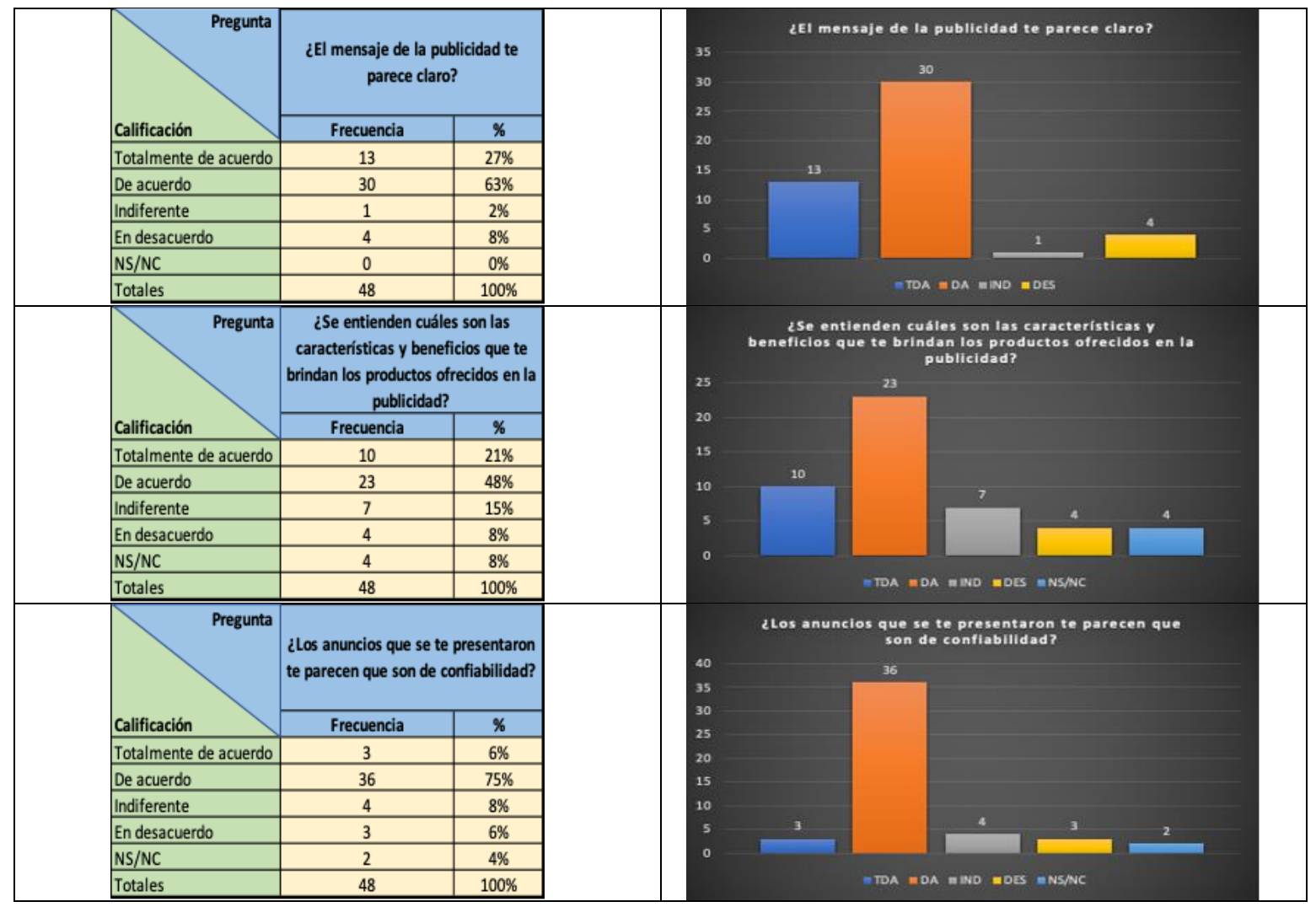

